\title{
Metronome Use Positively Affect Cardiopulmonary Resuscitation Parameters: Prospective Observational Multicenter Manikin Study
}

\author{
Necmiye Yalcin Ocak ${ }^{1}$, Mustafa Sever ${ }^{1, \star}$, Orkun Unek ${ }^{1}$, \\ Ibrahim Toker ${ }^{1}$, Emirhan Erdem ${ }^{1}$, Murat Yesilaras ${ }^{1}$, \\ Ozge Duman Atilla ${ }^{1}$
}

\author{
${ }^{1}$ Department of Emergency Medicine, \\ Tepecik Education and Research \\ Hospital, Izmir, Turkey

\section{*Correspondence} \\ adanasever@yahoo.com (Mustafa Sever)
}

\begin{abstract}
Objective: Clinical studies have shown that eliminating performer errors is important to ensure high quality cardiopulmonary resuscitation (CPR). Literature on the effects of metronome use on the quality of CPR is scarce. This study aimed to investigate the effect of metronome use on the quality of cardiopulmonary resuscitation. Methods: Thirty volunteer emergency physicians who were divided into 15 groups participated in this prospective, observational, multicenter, manikin study. Firstly, each participant performed conventional CPR on a manikin, and then performed metronome-guided CPR after a short break. Parameters affecting CPR quality were evaluated based on the recommendations of the 2015 American Heart Association CPR and Emergency Cardiovascular Care Guideline. In addition, the fatigue levels of participants were evaluated using the Borg Fatigue Index. Results: Metronome-guided CPR significantly improved the chest compression rate (median (Interquartile Range-IQR); 128 (22) compressions/min vs. 110 (2) compressions/min; 95\%CI, p $<0.001$ ), deep compression rate (median (IQR); 95.25 (80) compressions/min vs. 72.63 (105) compressions/min; 95\% CI, $\mathrm{p}<0.001$ ), compression depth (median (IQR); 62.50 (11) $\mathrm{mm}$ vs. 60.25 (14) $\mathrm{mm}$; 95\%CI, $\mathrm{p}=0.016$ ), ventilation number (median (IQR); 11.25 (6) ventilations/ $\mathrm{min}$ vs. 9.50 (1) ventilations $/ \mathrm{min} ; 95 \% \mathrm{CI}, \mathrm{p}=$ 0.001 ), high-volume ventilation count (median (IQR); 10.13 (6) ventilations/min vs. 9.50 (1) ventilations $/ \mathrm{min} ; 95 \% \mathrm{CI}, \mathrm{p}=0.026)$, minute ventilation volume (median (IQR); 11.75 (10) L/min vs. 8.03 (3) L/min; 95\%CI, p < 0.05), and fatigue levels (median (IQR); 3 (2) vs. $2(2)$; in $95 \% \mathrm{CI}, \mathrm{p}<0.05$ ). Conclusions: Our study showed that metronome is a useful device for reaching effective CPR. Metronome guidance may change the CPR parameters positively. This study is in accordance with previous studies which have investigated the effect of metronome-guided CPR on survival.
\end{abstract}

\section{Keywords}

Cardiopulmonary resuscitation, Metronome, Prospective study, Observational study, Multicenter study, Manikin study

\section{Introduction}

Cardiac arrest is one of the leading health problems in the world. High-quality cardiopulmonary resuscitation (CPR) is crucial because it is associated with a four-fold increase in the survival rate of cardiac arrest victims [1-4]. In the 2015 American Heart Association (AHA) guidelines update for CPR and Emergency Cardiovascular Care (ECC) pub- 
lished by the International Liaison Committee on Resuscitation (ILCOR), ensuring appropriate chest compressions of adequate depth and rate and avoiding hyperventilation have been emphasized as the cornerstones of high-quality CPR.In addition, it has been stated that although feedback devices which aim to improve the quality and ensure the standardization of CPR have been implemented into daily practice, these devices do not affect survival rates [4].

Studies investigating the use of a metronome to increase the quality and effectiveness of CPR have also been conducted [5-18]. The use of a metronome was shown to be effective in reaching the target compression [6-10, 14, 18] and ventilation rates [5-16], and to affect compression depth [6, 8-10, 12-15]. However, no definitive conclusions have been reached regarding the effects of metronome use on chest compression and ventilation rates [6-18].

The purpose of this study was to investigate the efficacy of metronome use on chest compression and ventilation rates in an intubated manikin model.

\section{Methods}

\subsection{Study design}

This was a multi-center, prospective, randomized, crossover, manikin-based, two-rescuer CPR simulation study. The study was conducted between October 21, 2015, and December 1, 2015, in the emergency medicine departments of Izmir Tepecik Education and Research Hospital (Center 1), Ege University Hospital (Center 2), and 9 Eylul University Hospital (Center 3). Each of these departments receive about 150-200 adult out-ofhospital cardiac arrest cases annually. Before starting the study, ethical approval was obtained from the local ethics committee (Tepecik Educational and Research Hospital Local Ethical Committee, Izmir; June 30 ${ }^{t h}, 2015$ - No:5).

\subsection{Equipment}

The AMBU ${ }^{\circledR}$ Adult Advanced Life Support (ALS) Training Manikin was used to assess the CPR performance of participants. Using the "StudioOne 3.3.3.41198 Win x64" program, a melodic rhythm consisting of two different sounds for compression and ventilation was created. The rhythm was determined based on the 2015 AHACPR guidelines, with a compression rate of $110 / \mathrm{min}$ and a ventilation rate of 10 breaths/min [3]. (Appendix: Metronome guidance rhythm audio file)

\subsection{Study setting}

In order to standardize ventilation during CPR, all manikins were intubated with a size 8 Portex $^{\circledR}$ endotracheal intubation tube. The endotracheal tube was taped at $22 \mathrm{~cm}$. A standard bag-valve device $\left(\mathrm{AMBU}^{\circledR} 1.6 \mathrm{~L}\right)$ was used for ventilation. The manikin was placed on a CPR board on a stretcher. The height of the stretcher was adjusted according to each participant. In order to create a realistic simulation, the chest stiffness of the manikin was adjusted to the highest level.

The periodic ventilation and chest compression skills of each participant were recorded using the built-in software feature of the manikin (AMBU ${ }^{\circledR}$ manikin software). This software enables saving each compression and ventilation data derived from the manikin so that they can be assessed individually. All manikins were calibrated prior to each group. The fatigue levels of participants were evaluated using the 10-step "Modified Borg Fatigue Scale" (MBFS) $[19,20]$. The evaluation was repeated at the end of each 2-minute cycle.

\subsection{Selection of participants}

Emergency medicine residents with more than 6 months of professional experience who had previously received training on basic and advanced cardiac life support and who volunteered to participate in the study were recruited. The simulation experiments were carried out in three different centers. In order to render the conditions similar, the experiments were conducted in the resuscitation unit of each center. The participants were divided into groups of two. Each participant performed 2 sets of CPR: 8 minutes of conventional CPR and 8 minutes of metronomeguided CPR. The 8 minute-duration was chosen to create a moderate level of fatigue.

The participants switched places every 2 minutes during the 8-minute CPR. After conventional CPR was complete, a 30-minute break was given before metronome-guided $\mathrm{CPR}$ in order to avoid fatigue. In this break, the audible metronome melody was introduced to the participants.

\subsection{Choosing the reference standard}

In this simulation CPR study, the parameters recommended by the AHA CPR and ECC Guidelines were used as the reference standard. Thus, a compression rate of $110 / \mathrm{min}$, compression depth of $50-60 \mathrm{~mm}$, ventilation rate of $10 / \mathrm{min}$, tidal volume of 0.5-0.6 L, and minute ventilation of 1.5-6 $\mathrm{L} / \mathrm{min}$, with the hands positioned on the lower half of the sternum were deemed acceptable.

A shallow compression was defined as a chest compression depth of less than $50 \mathrm{~mm}$, and a deep compression was defined as a chest compression depth of greater than $60 \mathrm{~mm}$. A ventilation with a tidal volume of less than $0.5 \mathrm{~L}$ was defined as a low-volume ventilation, and a ventilation with a tidal volume of greater than $0.6 \mathrm{~L}$ was defined as a highvolume ventilation. Allowing the chest to return to its initial (static) state after each chest compression was defined as "full chest recoil".

The data on compression rate, compression depth, full chest recoil, ventilation rate, mean tidal volume, mean minute ventilation and incorrect hand position were recorded by the manikin software program as graphics and mean values. 
TA B L E 1. Chest compression parameters during cardiopulmonary resuscitation.

\begin{tabular}{lccc} 
Compression Parameters & $\begin{array}{c}\text { Conventional CPR } \\
(\text { mean } \pm \text { SD) }\end{array}$ & $\begin{array}{c}\text { Metronome-guided CPR } \\
(\text { mean } \pm \text { SD) }\end{array}$ & p-value \\
\hline Compression rate $(/ \mathrm{min})$ & $130 \pm 14.1$ & $110.9 \pm 1.5$ & $<0.001$ \\
\hline Compression depth $(\mathrm{mm})$ & $62.1 \pm 6.7$ & $59.3 \pm 8.6$ & 0.016 \\
\hline Shallow compressions $(/ \mathrm{min})$ & $13.8 \pm 23.1$ & $21.5 \pm 33.9$ & 0.297 \\
\hline Deep compressions $(/ \mathrm{min})$ & $81 \pm 44.2$ & $59.8 \pm 45.5$ & 0.006 \\
\hline Optimal compressions $(/ \mathrm{min})$ & $34.3 \pm 35.4$ & $29.1 \pm 27.7$ & 0.339 \\
Compressions with incomplete release $(/ \mathrm{min})$ & $45.4 \pm 52.5$ & $35.8 \pm 43.8$ & 0.174 \\
\hline
\end{tabular}

M: Median; IQR: Interquartile Range.

\subsection{Statistical analysis}

All statistical analyses were performed using the Statistical Package for Social Sciences ${ }^{\circledR}$ (SSPS) software (IBM SPSS for Windows, Version 22, SPSS Inc., USA). The distribution of the data was analyzed using the ShapiroWilk Normality test. The data were not normally distributed, so the Wilcoxon Signed Ranks test was used for comparison. Quantitative variables were presented as median and interquartile range (IQR), and qualitative variables were expressed as numbers and percentages. Based on the mean compression rate values in secured airway scenario reported by Kern et al, the sample size was calculated to be 28 participants with a $95 \%$ confidence interval and $80 \%$ power [7]. Considering the margin of error, 30 participants were included. All analyses were performed within a $95 \%$ confidence interval and a $p$-value $<0.05$ was considered statistically significant.

\section{Results}

The number of emergency medicine residents working in centers 1,2 , and 3 was 24,15 , and 16 , respectively. The number of residents who volunteered to participate in the study were $17(56.7 \%), 6(20 \%)$, and $7(23 \%)$ for Centers 1,2 , and 3 , respectively. Of the participants, $19(63.3 \%)$ were male, and $11(36.7 \%)$ were female. The mean age was $30 \pm 2.9$ years.

For each method, the compression rate according to compression depth and the compression rate with respect to the number of compressions without full chest recoil were calculated.

Compared to the conventional CPR group, the median number (interquartile range; IQR) of chest compressions per minute (128 (22) vs $110(2), \mathrm{p}<0.001)$ and the median (IQR) chest compression depth 62.50 (11) vs 60.25 (14) $\mathrm{mm}, \mathrm{p}=0.016)$ decreased to within the optimal range in the metronome-guided CPR group. In addition, the number of shallow compressions increased by $8.8 \%(10.6 \%$ vs $19.4 \%$ ), the number of deep compressions decreased by $8.4 \%$ (62.3\% vs $53.9 \%)$, the number of compressions with optimal depth did not change ( $26.4 \%$ vs $26.2 \%)$, and the number of compressions without full chest recoil decreased by $2.7 \%$ (34.9\% vs $32.2 \%)$ in the metronome-guided CPR group.
The numbers of low-volume and high-volume ventilations were compared to the total number of ventilations for both groups. In the metronome-guided CPR group, the median number (IQR) of ventilations per minute decreased to within the optimal range (11.25 (6) vs 9.50 (1), p < $0.001)$, the number of low-volume ventilations decreased by $8.5 \%$ (14.8\% vs $6.3 \%)$ and the number of high-volume ventilations increased by $6.7 \%(78.5 \%$ vs $85.2 \%)$ in comparison to the conventional CPR group.

The median (IQR) minute ventilation volume decreased significantly in the metronome-guided CPR group (11.75 (10) vs 8.03 (3), p $<0.001$ ).

Hand-position errors were not observed in both CPR group $(\mathrm{p}=0.574)$.

The compression and ventilation parameters obtained during metronome-guided $\mathrm{CPR}$ and conventional $\mathrm{CPR}$ are presented in Tables 1 and 2, respectively.

The median (IQR) fatigue levels measured according to the MBFS were significantly lower in the metronomeguided CPR group compared to the conventional CPR group (2 (2) vs 3 (2), respectively).

\section{Discussion}

With the metronome-guided two-rescuer CPR model used in our study, the target compression and ventilation rates recommended by the current guidelines were achieved. In addition, we observed that minute ventilation decreased significantly, even though the target range could not be attained. To our knowledge, this parameter has not been investigated before in the literature.

In CPR simulation studies carried out on untrained bystanders by Rasmussen [14] and Park [8] and in those carried out on trained rescuers by Zimmerman [10], Hafner [9],Chung [18], Kern [7] and Jantti [6], the use of a metronome during CPR was found to increase success rates in terms of reaching target chest compression rates (p-values; 0.04, 0.018, 0.001, 0.002, 0.001, 0.001, and $<$ 0.001 , respectively).

In studies on trained rescuers, compression rate was found to be higher in the conventional CPR group compared to the metronome-guided CPR group. On the contrary, in studies on untrained laypersons, compression rate was found to be higher in the metronome-guided CPR group 
TA B L E 2. Ventilation parameters during cardiopulmonary resuscitation.

\begin{tabular}{lccc} 
Ventilation Parameters & $\begin{array}{c}\text { Conventional CPR } \\
\text { (mean } \pm \text { SD) }\end{array}$ & $\begin{array}{c}\text { Metronome-guided CPR } \\
\text { (mean } \pm \text { SD) }\end{array}$ & p-value \\
\hline Number of ventilations (/min) & $13.5 \pm 6.6$ & $9.5 \pm 0.5$ & 0.001 \\
\hline Low-volume ventilations (/min) & $2 \pm 5.3$ & $0.6 \pm 1.3$ & 0.086 \\
\hline High-volume ventilations (/min) & $10.6 \pm 7.4$ & $8.1 \pm 2.9$ & 0.026 \\
Ventilation volume (Liters) & $0.8 \pm 0.3$ & $0.8 \pm 0.2$ & 0.879 \\
\hline Minute ventilation (Liters) & $13.6 \pm 7.6$ & $9.1 \pm 5.2$ & 0.001 \\
\hline
\end{tabular}

M: Median; IQR: Interquartile Range.

compared to the conventional CPR group.

On the other hand, literature investigating the effect of metronome use on compression depth has reached different conclusions [6, 8-10, 12-15]. In the studies by Park [8], Hafner [9] and Zimmerman [10], it was found that even though metronome use caused a decrease in chest compression depth, it did not have a significant effect on reaching the target values. Jantti et al [6] have also reported a decrease in compression depth. In opposition, Chung [12, 13], Rasmussen [14] and Bae [15] have reported an increase in compression depth.

All of these studies have concluded that compression depth is affected by compression rate. The fact that the compression depth has either stayed the same or decreased in studies where the metronome rhythm used during CPR was equal to or slower than the rate previously learned by the trained rescuer supports this conclusion [6, 9, 10]. Similarly, the fact that compression depth was reported to increase in studies where the metronome rhythm used during CPR was faster compared to the rate the trained rescuer had previously learned also supports this conclusion [12, 13, 15].

In our study, the chest compression rate achieved by trained rescuers during conventional CPR was higher than the target range. On the other hand, in the metronomeguided CPR group, both chest compression rate and chest compression depth decreased to within the target range. This finding is in accordance with the conclusion mentioned above. In our study, the number of deep compressions was also found to decrease significantly with the use of a metronome. We believe that this result stems from the fact that the rescuers performed faster and deeper compressions during conventional CPR due to the "push hard, push fast" instruction they received during their previous CPR training.

In our opinion, metronome use affects compression depth by correcting the compression rate. In our study, metronome use was found to decrease compression depth and slightly increase the number of shallow compressions, but this increase was not significant. Similar to our study, Park et al [8]. found that metronome use increased the number of shallow chest compressions. However, in contrast to our study, this increase was found to be significant. This difference may be due to the fact that Park et al. recruited untrained participants in their study.
In accordance with the similar studies in the literature $[8,10,15,18]$, we found that metronome use had no effect on ensuring full chest recoil or correct hand position. Rasmussen et al [14]. reported that metronome use caused a significant decrease in incorrect hand position rates. We believe that this difference was due to the fact that rescuers in the metronome-guided and conventional CPR groups were trained separately in terms of hand positioning.

\subsection{Ventilation rate and volume}

In their studies on the effect of metronome use on ventilation during CPR, Kern and Chung reported that metronome use prevented hyperventilation by keeping the ventilation rate within the range recommended in the guidelines [7, 18].

$\mathrm{Na}$ et al. [16], adopted to the 30:2 compression to ventilation ratio, performed ventilations according to different compression rates (e.g. 100, 110, 120 compressions/min). They measured the mean airway pressure and tidal volume in order to assess the quality of ventilation. They reported that as the speed of the metronome rhythm increased, the quality of ventilation decreased. In addition, they recommended a chest compression rate of $100 / \mathrm{min}$ in case of a respiratory etiology [16].

Both in the out-of-hospital study by Aufderheide [5] and in the in-hospital study by Milander et al. [11], trained rescuers were found to perform significant hyperventilation which compromised hemodynamic and pulmonary functions and decreased the survival rates.

As stated by Aufderheide et al. [5], the impact of hyperventilation on survival is not related to a decrease in the coronary perfusion pressure caused by hypocapnia. The physiological outcomes of hyperventilation are an increase in positive intrathoracic pressure during the decompression phase of CPR, a decrease in cardiac preload and output, and a reduction in right ventricular function. An increase in the tidal volume is also known to affect cardiac output negatively. This whole process leads to a decrease in survival rates [5].

The above-mentioned studies have emphasized that despite all the training they received, trained rescuers still hyperventilated patients and that the training programs were insufficient in preventing hyperventilation $[5,11]$.

The present study is the first objective simulation study performed using a metronome with different sound alerts for compression and ventilation. It differs from previous 
studies in that ventilation was simulated through an advanced airway. The metronome model used in our study succeeded in ensuring that the ventilation rate is within the range recommended in the guidelines. This way, ventilation rate was lowered without causing a change in the tidal volume, resulting in a decrease in minute ventilation and in hyperventilation. This seems to be an effective solution for hyperventilation which has a negative effect on survival as pointed out by Aufderheide et al.

Even though the metronome-guided CPR model used in our study managed to yield minute ventilation values close to the ones recommended in the guidelines, these values are still slightly above the target. This stems from the fact that the mean ventilation volume was high in both groups. The high ventilation volume was attributed to the fact that ventilation was performed by the same participant using the same bag-valve device.

\subsection{Fatigue}

Chung [18] and Jantti [6] reported that metronome use had no effect on fatigue levels. When the CPR duration was set at 8 minutes to induce a moderate level of fatigue, it was observed that metronome use could decrease the fatigue levels of rescuers, even if the chest wall stiffness of the manikin was adjusted to the highest level. This was attributed to the fact that compression rate, compression depth, and ventilation rate were higher in the conventional CPR group compared to the metronome-guided CPR group, which caused the rescuers in the conventional CPR group to exert more effort.

\section{Conclusion}

The metronome, which has a melodic rhythm consisting of two different sounds for compression and ventilation, is an effective tool for reaching the target compression rate by decreasing hyperventilation and optimizing compression depth without causing fatigue. Metronome use may be an easy and cost-effective way to change CPR parameters positively.

\section{Limitations}

Due to the technical characteristics of the device used, the recordings could be made only at 2-minute intervals. At the end of each cycle, short breaks of about 10 seconds had to be taken in order for the participants to swap places for compression and ventilation. This has caused a pause in the CPR cycle.

\section{ACKNOWLEDGMENTS}

Authors thanks to Dr Ece Akceylan for linguistic support and also thanks to $\mathrm{PhD}$ Asli Suner for statistical support.

\section{CONFLICT OF INTEREST}

We declare that we do not have any commercial or associative interest that represents a conflict of interest in connection with the work submitted.

\section{REFERENCES}

[1] Gallagher EJ, Lombardi G, Gennis P. Effectiveness of bystander cardiopulmonary resuscitation and survival following out-of-hospital cardiac arrest. JAMA. 1995;274:1922-5.

[2] Van Hoeyweghen RJ, Bossaert LL, Mullie A, Calle P, Martens P, Buylaert WA, et al. Quality and efficiency of bystander CPR. Belgian Cerebral Resuscitation Study Group. Resuscitation 1993;26:47-52.

[3] Kleinman ME, Brennan EE, Goldberger ZD, Swor RA, Terry M, Bobrow BJ, et al. Part 5: Adult Basic Life Support and Cardiopulmonary Resuscitation Quality: 2015 American Heart Association Guidelines Update for Cardiopulmonary Resuscitation and Emergency Cardiovascular Care. Circulation 2015;132:414-435.

[4] Yannopoulos D, Aufderheide TP, Gabrielli A, Beiser DG, McKnite $\mathrm{SH}$, Pirrallo RG, et al. Clinical and hemodynamic comparison of 15:2 and 30:2 compression-to-ventilation ratios for cardiopulmonary resuscitation. Crit Care Med. 2006;34:1444-9.

[5] Aufderheide TP, Sigurdsson G, Pirrallo RG, Yannopoulos D, McKnite $\mathrm{S}$, von Briesen $\mathrm{C}$, et al. Hiperventilation-induced hypotension during cardiopulmonary resuscitation. Circulation. 2004;109:1960-5.

[6] Jäntti H, Silfvast T, Turpeinen A, Kiviniemi V, Uusaro A. Influence of chest compression rate guidance on the quality of cardiopulmonary resuscitation performed on manikins. Resuscitation. 2009;80:453-7.

[7] Kern KB, Stickney RE, Gallison L, Smith RE. Metronome improves compression and ventilation rates during $\mathrm{CPR}$ on a manikin in a randomized trial. Resuscitation. 2010;81:206-10.

[8] Park SO, Hong CK, Shin DH, Lee JH, Hwang SY. Efficacy of metronome sound guidance via a phone speaker during dispatcherassisted compression-only cardiopulmonary resuscitation by an untrained layperson: a randomised controlled simulation study using a manikin. Emerg Med J. 2013;30:657-61.

[9] Hafner JW, Jou AC, Wang H, Bleess BB, Tham SK. Death before disco: the effectiveness of a musical metronome in layperson cardiopulmonary resuscitation training. J Emerg Med. 2015;48:4352. Zimmerman E, Cohen N, Maniaci V, Pena B, Lozano JM, Linares M. Use of a Metronome in Cardiopulmonary Resuscitation: A Simulation Study. Pediatrics. 2015;136:905-11.

[10] Zimmerman E, Cohen N, Maniaci V, Pena B, Lozano JM, Linares M. Use of a Metronome in Cardiopulmonary Resuscitation: A Simulation Study. Pediatrics. 2015;136:905-11.

[11] Milander MM, Hiscok PS, Sanders AB, Kern KB, Berg RA, Ewy GA. Chest compression and ventilation rates during cardiopulmonary resuscitation: the effects of audible tone guidance. Acad Emerg Med. 1995;2:708-13.

[12] Chung TN, Kim SW, You JS, Cho YS, Chung SP, Park I. A higher chest compression rate may be necessary for metronome-guided cardiopulmonary resuscitation. Am J Emerg Med. 2012;30:226-30.

[13] Chung TN, Bae J, Kim EC, Cho YK, You JS, Choi SW, et al. Induction of a shorter compression phase is correlated with a deeper chest compression during metronome-guided cardiopulmonary resuscitation: a manikin study. Emerg Med J. 2013;30:551-4.

[14] Rasmussen SE, Nebsbjerg MA, Krogh LQ, Bjørnshave K, Krogh $\mathrm{K}$, Povlsen JA, et al. A novel protocol for dispatcher assisted CPR improves $\mathrm{CPR}$ quality and motivation among rescuers-A randomized controlled simulation study. Resuscitation. 2017;110:74-80.

[15] Bae, J, Chung TN, Je SM. Effect of the rate of chest compression familiarised in previous training on the depth of chest compression during metronome-guided cardiopulmonary resuscitation: a randomised crossover trial. BMJ Open. 2016;6:e010873.

[16] Na JU, Han SK, Choi PC, Shin DH. Effect of metronome rates on the quality of bag-mask ventilation during metronome-guided 
30:2 cardiopulmonary resuscitation: A randomized simulation study. World J Emerg Med. 2017;8:136-140.

[17] Lee CJ, Chung TN, Bae J, Kim EC, Choi SW, Kim OJ. 50\% duty cycle may be inappropriate to achieve a sufficient chest compression depth when cardiopulmonary resuscitation is performed by female or light rescuers. Clin Exp Emerg Med. 2015;2:9-15.

[18] Chung TN, Kim SW, You JS, Cho YS, Chung SP, Park I, et al. The specific effect of metronome guidance on the quality of oneperson cardiopulmonary resuscitation and rescuer fatigue. J Emerg Med. 2012;43:1049-54.

[19] Mahler DA, Horowitz MB. Clinical evaluation of exertional dyspnea. Clin Chest Med. 1994;15:259-69.
[20] Ries AL, Bauldoff GS, Carlin BW, Casaburi R, Emery CF, Mahler DA, et al. Pulmonary Rehabilitation: Joint ACCP/AACVPR Evidence-Based Clinical Practice Guidelines. Chest. 2007;131:4S$42 \mathrm{~S}$.

How to cite this article: Necmiye Yalcin Ocak, Mustafa Sever, Orkun Unek, et al. Metronome Use Positively Affect Cardiopulmonary Resuscitation Parameters: Prospective Observational Multicenter Manikin Study. Signa Vitae. 2020;16(1):33-38. doi:10.22514/sv.2020.16.0005. 